\title{
Resilienz vs. Effizienz - ein kritischer Blick auf die Umweltökonomie
}

\author{
Dirk Löhr
}

Erhalten: 2. März 2009/Akzeptiert: 15. März 2009/ Online veröffentlicht: 31. März 2009

(C) Springer-Verlag 2009

Zusammenfassung Ziel und Hintergrund Ökonomen beurteilen sämtliche (umwelt-)politische Maßnahmen - darunter auch die Bereitstellung von Energie - vor allem unter Effizienzgesichtspunkten. So sollen ökonomische Instrumente des Umweltschutzes in (kosten-)effizienter Weise dazu beitragen, Umweltziele zu erreichen. Es wird jedoch dargestellt, dass die für die Ökonomie charakteristische Überbetonung des Effizienzkriteriums eine den ökologischen Systemen fremde Logik ist. Auch ökologische Systeme sind oftmals nicht hochgradig effizient. In Gestalt ökonomischer Instrumente des Umweltschutzes kann die Übertragung der Effizienzlogik auf natürliche Systeme aber u. a. dazu beitragen, die Resilienz von Ökosystemen zu gefährden. Dies wird am Beispiel des Emissionshandels illustriert.

Zeiteffizienz wird v. a. vor dem Hintergrund der Renditeerfordernisse der Anleger zu einem bedeutsamen Beurteilungskriterium auch bei der Durchführung von Öko-Investitionen. Der Beitrag stellt dar, wie die Fokussierung auf das Renditeerfordernis zu Einförmigkeit führt, was ebenfalls negative Auswirkungen auf die Resilienz von (Öko-)Systemen haben kann.

Schwerpunkte Das Wirtschaftsystem ist eingebettet in Gesellschaft und natürliche Umwelt, die aus (sich) selbstorganisierenden (,lebenden') Systemen bestehen. Die Wirtschaft als instrumentelles System sollte diesen gegenüber eine dienende Funktion haben. Die systemare Leitwerttheorie gibt Anhaltspunkte dafür, dass die Überbetonung eines Leitwerts (wie dies durch die Ökonomie in Gestalt des domi-

Verantwortliche Herausgeber: Martin Pehnt · Eckard Helmers

Dirk Löhr ( $ه)$

Umwelt-Campus Birkenfeld, Postfach 1380,

55761 Birkenfeld, Deutschland

E-Mail: d.loehr@umwelt-campus.de nanten Effizienzkriteriums geschieht) zur Schwächung der Lebens- und Entwicklungsfähigkeit gesellschaftlicher und ökologischer Systeme bis hin zu deren Zusammenbruch führen kann.

Ergebnisse und Diskussion Der Beitrag zielt auf eine Veränderung der Perspektive, die wahrscheinlich nur von außen in die ökonomische Fachwissenschaft getragen werden kann. Die auf das Effizienzparadigma fokussierte Umweltökonomie kann auf die drängenden ökologischen Probleme zurzeit keine befriedigende Antwort geben.

Schlussfolgerungen Wenn vorliegend das Primat des Effizienzkriteriums infrage gestellt wird, soll nicht die Ausblendung dieses Kriteriums propagiert werden. Vielmehr richten wir uns gegen die einseitige, nicht systemare, die Leitwerttheorie missachtende Dominanz des Leitwertes Effizienz.

Ausblick Wird das ,Effizienzparadigma' nicht mehr unbesehen akzeptiert, so sind einige ,heilige Kühe" - wie der Emissionshandel oder die Orientierung am Shareholder Value grundsätzlich zu überdenken und alternative Designs zu entwickeln.

Schlüsselwörter Effizienz · Resilienz · Emissionshandel · Leitwerttheorie $\cdot$ Vielfalt

\section{Resilience vs. efficiency - a critical view on the contemporary environmental economic science}

Abstract Background, aim, and scope Economists assess politics (also concerning energy supply) due to the efficiency criterion. Thus, economic instruments for environmental protection shall contribute to achieve ecological goals in an (cost-)efficient way. We show that the overemphasis of efficiency is an alien in the way (ecological) systems are working. Mostly, ecological systems are not high-grade efficient. 
Hence, economic instruments of environmental protection introduce an inappropriate logic to ecological systems that may have severe impacts on their resilience. We illustrate this with the example of emission trading.

Time efficiency is considered to be a powerful criterion due to the interest yield requirements of investors, also for ecological investments. We show how the concentration on time efficiency destroys diversity and has negative impacts on the resilience of (eco-)systems.

Main features The economic system is embedded in society and natural environment (as self-organizing, living systems). The economy as an 'instrumental system' should serve these systems. The guiding value approach (a system theory approach) gives indication that overemphasizing certain guiding values (such as efficiency, as the economic science does) may weaken the functional capability of systems and finally may lead to a collapse of the systems.

Results and discussion The article tries to change the focus of the discussion. An altered focus probably has to be implemented by scientists of other subject areas. Contemporary environmental economics (with its focus on efficiency) is unable to give satisfying answers on the pestering problems.

Conclusions Criticising the primacy of efficiency is not the same as generally to disclaim considering efficiency needs. Instead, based on the guiding value theory, we want to be contrary to the one-sided and dominating stressing of the of the efficiency criterion.

Perspectives Not following the efficiency guide any more means to think over some 'sacred cows' such as emission trading or shareholder value. Instead we have to think over alternative designs to reach the ecological targets.

Keywords Diversity · Efficiency · Resilience · Emission trading $\cdot$ Guide value theory

\section{Problemstellung}

Das vorrangige Kriterium zur Beurteilung von politischen Maßnahmen ist für Ökonomen die Effizienz. Dies gilt auch für energiepolitische Maßnahmen im weitesten Sinne. Unter Effizienz wird in der Wirtschaftswissenschaft einerseits (v. a. auf gesamtwirtschaftlicher Ebene) Allokationseffizienz verstanden: Hiernach soll der gesamtgesellschaftliche Überschuss der Nutzen über die Kosten maximiert werden. Dieser gesellschaftliche Optimalpunkt kann bei Bestehen (negativer) externer Effekte verfehlt werden. Bei externen Effekten handelt es sich um die unkompensierten Auswirkungen ökonomischer Entscheidungen auf unbeteiligte Marktteilnehmer, die nicht in das Entscheidungskalkül der Verursacher eingehen. Der Verursacher einer Emission kann beispielsweise billiger produzieren und daher eine größere Menge seines Produktes absetzen, wenn er auf Vermeidungsanstrengungen verzichtet. Den Schaden der Emission - auch aus der Energiebereitstellung - tragen andere, z. B. durch Gesundheitsbeeinträchtigungen. Volkswirtschaftlich werden externe Effekte als eine Ursache für Marktversagen gesehen, woraus sich die Notwendigkeit staatlicher Regulierung ergibt. Dies ist das traditionelle Terrain, auf dem sich die Umweltökonomie bewegt. Neben der Allokationseffizienz wird aus einer zumeist eher einzelwirtschaftlichen bzw. politisch-praktischen Perspektive Kosteneffizienz propagiert. Liegt gleichermaßen Kosteneffizienz und allokative Effizienz vor, spricht man auch von ,Markteffizienz' ${ }^{1}$ Unter einer anderen - außerökonomischen und damit breiteren Perspektive - kann Effizienz einmal als Ressourceneffizienz (wobei wir diese in einem weiteren Sinne verstehen wollen, indem sie nicht nur die Nutzung von Ressourcen, sondern auch die der Deponien und Senken umfasst) und Zeiteffizienz betrachtet werden (Müller-Reißmann 1996). Als markt- und ressourceneffiziente Instrumente des Umweltschutzes werden v. a. Ökosteuern und Emissionszertifikate diskutiert. Nachfolgend wird dargestellt, warum den marktwirtschaftlichen Instrumenten des Umweltschutzes ein schwerer Pferdefuß anhaftet: Sie neigen nämlich angesichts ihrer einseitigen Fokussierung auf Effizienz dazu, die Resilienz (also die Fähigkeit eines Systems, seine Selbstorganisation auch angesichts externer Störungen zu erhalten) von ökologischen (Sub-)Systemen nicht hinreichend zu beachten.

Schließlich wird auf den Aspekt der Zeiteffizienz eingegangen. Hierbei wird die Rolle von Zins und Rentabilitätsprinzip diskutiert, die zu einer Verringerung der Vielfalt von Formen und Arten sowie zu einer Beschleunigung des Lebens führt. Dies drückt sich auch und gerade in der gegenwärtigen Bevorzugung fossiler Energien aus.

Im Rahmen der Systembetrachtung greifen wir auf die Leitwerttheorie zurück (Müller-Reißmann et al. 1988). Hiernach muss sich jedes ,selbstorganisierende' System (Lebewesen, Unternehmen, Stadt, Gesellschaft, etc.) an bestimmten Leitwerten (Effizienz, Sicherheit, Handlungsfreiheit, Adaptivität, physisch-psychische Existenz- und Reproduktionsbedingungen, Gerechtigkeit/Verantwortung/ Solidarität) orientieren. Sowohl die Überbetonung als auch die Außerachtlassung eines Leitwertes kann die Funktionsfähigkeit des ökonomischen bzw. die Lebens- und Entwicklungsfähigkeit des ökologischen Gesamtsystems gefährden. Wir wollen dies anhand der Fokussierung der herrschenden (Umwelt-)Ökonomie auf den Leitwert der Effizienz illustrieren und plädieren für eine Relativierung der Bedeutung dieses Leitwertes.

\footnotetext{
${ }^{1}$ Markteffizienz impliziert damit zugleich Pareto-Effizienz bzw. das Vorliegen des Kaldor-Hicks-Kriteriums (vgl. Borrmann und Finsinger 1999).
} 
Am Ende des Artikels wurde noch ein Glossar hinzugefügt, in dem ergänzend zum Text einige wichtigste Fachausdrücke erläutert werden.

\section{Ressourceneffizienz und Resilienz - am Beispiel des Emissionshandels}

Das von der herkömmlichen Ökonomik eingeforderte Primat der Effizienz kommt in besonderer Weise in den marktwirtschaftlichen Instrumenten des Umweltschutzes zum Ausdruck $^{2}$, die zunehmend propagiert werden, um u.a. die klimaschädigenden Treibhausgase, welche v. a. durch die Energieproduktion und -nutzung entstehen, besser in den Griff zu bekommen. Dabei geht es v.a. um die Ausschöpfung von Kosteneffizienz (bzw. Ressourceneffizienz, vgl. oben). Um die Effizienz möglichst gut ausschöpfen zu können, sind marktwirtschaftliche Instrumente des Umweltschutzes auf ein großformatiges Design angewiesen: Man benötigt möglichst große Märkte mit vielen Marktteilnehmern. Warum dies jedoch gerade in ökologischer Hinsicht bedenklich sein kann, soll am Beispiel des Zertifikatehandels in der Ausgestaltung eines sog. ,Cap-and-Trade-Systems' illustriert werden.

\subsection{Zur Funktionsweise des Emissionshandels}

Die Umweltökonomie übertrug mit den ökonomischen Instrumenten des Umweltschutzes die Idee des Marktmechanismus mit Angebot und Nachfrage als maßgeblichen Bestimmungsgrößen auf die Umweltpolitik. Dabei bestimmen die sog. ,Grenzvermeidungskosten' die Nachfrage nach Verschmutzungsrechten: Grenzvermeidungskosten lassen sich einmal als die zusätzlichen Kosten interpretieren, die durch technische Vermeidung entstehen, andererseits als der Gewinn, auf den bei Produktionseinschränkung verzichtet werden muss (Opportunitätsgrenzkosten der Emissionsvermeidung). Das Wörtchen ,Grenz-' drückt also eine marginale Betrachtungsweise aus (mathematisch durch die erste Ableitung der betreffenden (Kosten-)Funktion dargestellt). Über die Grenzvermeidungskosten bestehen zumeist einigermaßen konkrete Vorstellungen bzw. valide Schätzungen. Als Grenzschadenskosten wird hingegen (ebenfalls in marginaler Betrachtung) derjenige zusätzliche (bewertete) Schaden bezeichnet, der bei der Produktion der letzten Einheit eines Gutes entsteht. Die Grenzschadenskostenkurve kann

\footnotetext{
${ }^{2}$ Das gilt nicht nur für die Internalisierungskonzepte, sondern auch für die Instrumente der standardorientierten Umweltpolitik - zumindest in der dominierenden Lesart. Allerdings spielt das Effizienzkriterium im Rahmen der standardorientierten Umweltökonomie nicht dieselbe Rolle wie bei den Internalisierungsstrategien, da sich die standardorientierte Umweltökonomie vom hehren Bezugspunkt des Wohlfahrtsoptimums verabschiedet hat.
}

entweder (je nach unterstellter Verteilung der Eigentumsrechte) als die Zahlungsbereitschaft eines Geschädigten für die Rückführung des Schadens oder die Zahlungsforderung des Geschädigten für die Duldung eines negativen externen Effektes interpretiert werden (Endres 2000).

Eine totale Vermeidung von Emissionen, die z.B. aus der Bereitstellung von Energie resultieren können, wird von der ökonomischen Theorie abgelehnt. Dies wäre nämlich nur unter Inkaufnahme unzumutbarer Belastungen möglich (im Extremfall die Einstellung jeglichen Wirtschaftens). Die Grenzschadenskosten wären dann zwar minimiert, die Grenzvermeidungskosten aber maximiert. Umgekehrt würden bei jeglichem Fehlen von Regulierung keine Vermeidungsanstrengungen vorgenommen: Die Grenzvermeidungskosten wären dann zwar minimal, aber der verursachte Grenzschaden maximal. In der ökonomischen Theorie sollte idealerweise der Schnittpunkt zwischen Grenzschadens(als Kosten der zusätzlichen Emissionen) und Grenzvermeidungskosten (als Nutzen der zusätzlichen Emissionen) angestrebt werden: Solange die Grenzvermeidungskosten (Emissionsnutzen) höher sind als die Grenzschadenskosten (Emissionskosten), sollte die schädigende Aktivität noch ausgedehnt werden. Bei Gleichstand wäre die schädigende wirtschaftliche Aktivität nicht mehr weiter auszudehnen (Abb. 1).

In der politischen Praxis verzichtet man allerdings auf den Versuch einer Ermittlung der Grenzschadenskosten und damit auf die Feststellung des ökonomischen Optimums v. a. deswegen, weil bezogen auf die Grenzschadenskosten (anders als bei den Grenzvermeidungskosten) wegen Bewertungsproblemen zumeist kaum konkrete Vorstellungen bestehen. Das Angebot an Verschmutzungsrechten wird vielmehr politisch - im Idealfall an naturwissenschaftlichen Erkenntnissen anstatt an politischen Kompromissen orientiert - festgelegt (sog. , standardorientierte Umweltpolitik'). Dies bedeutet z.B., dass für Emissionszertifikate ein Cap (Obergrenze) festgelegt wird, und zwar unabhängig von einer genauen Feststellung der Grenzschadenskosten. Bei diesem Cap-and-Trade-System wird also ein bestimmtes, begrenztes Angebot an Verschmutzungsrechten geschaffen. Im Idealfall korrespondiert dieses mit dem umweltökonomischen Optimalpunkt; das muss aber keinesfalls so sein und wird auch nicht unmittelbar angestrebt (Abb. 2).

Emissionen setzen die Inhaberschaft einer entsprechenden Menge von Verschmutzungsrechten voraus. Die Zertifikate werden handelbar gestellt. Diejenigen Marktteilnehmer, die in der Lage sind, Emissionen zu geringen Kosten zu vermeiden, können die nicht mehr benötigten Zertifikate auf dem Markt zum Marktpreis anbieten. Die Unternehmen hingegen, die hohe (Grenz-)Vermeidungskosten aufweisen, können die angebotenen Verschmutzungsrechte erwerben und die Vermeidung der Emission unterlassen. Sie stellen die Nachfrager auf dem Zertifikatemarkt dar. 
Abb. 1 Optimalpunkt in der umweltökonomischen Theorie
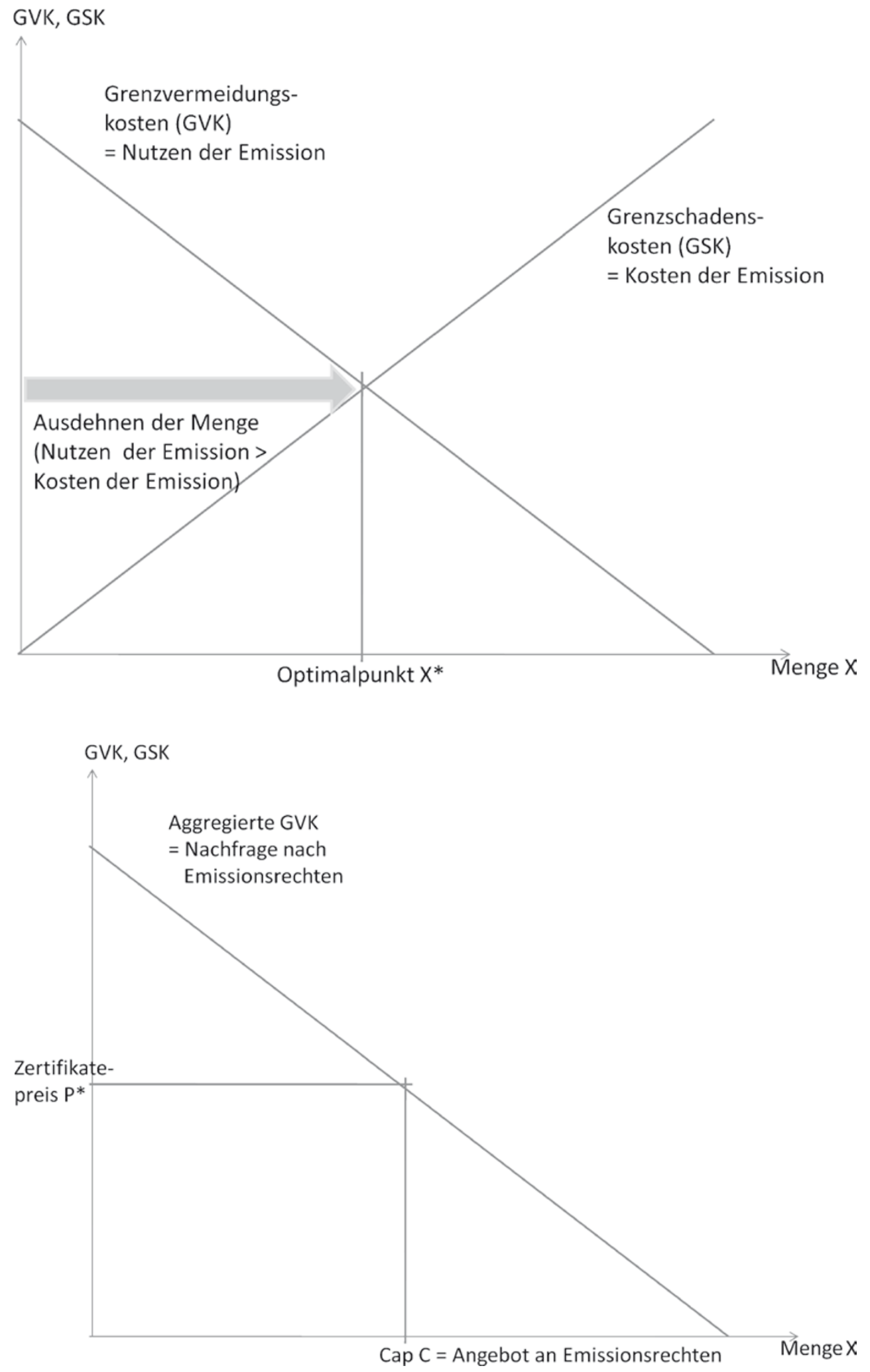

Abb. 2 Herausbildung des Zertifikatepreises im Cap-and-Trade-Regime
Die Kurve der aggregierten Grenzvermeidungskosten aller Verschmutzer ist identisch mit der Nachfragekurve nach den Zertifikaten. Beim Emissionshandel im Cap-and-Trade-
System ergibt sich der Zertifikatepreis dann im Schnittpunkt von Angebots- (Cap) und Nachfragekurve (aggregierte Grenzvermeidungskostenkurve). 


\subsection{Zur Bedeutung eines großformatigen Systems}

Der Emissionshandel funktioniert am besten vor dem Hintergrund eines großformatigen Designs,

- weil sich die Effizienz des Regimes aus den Unterschieden in den Grenzvermeidungskosten ergibt: Zumal diejenigen Unternehmen mit hohen Grenzvermeidungskosten zum Marktpreis Emissionszertifikate zukaufen, und solche mit geringen Grenzvermeidungskosten so lange ihre Emissionen verringern, wie ihre Grenzvermeidungskosten geringer als der Marktpreis sind (da sie zum Marktpreis veräußern und die Differenz zwischen Marktpreis und Grenzvermeidungskosten ihren Vermeidungsgewinn darstellt), gleichen sich die Grenzvermeidungskosten aller Unternehmen in einem effizienten Markt einander an: Sie entsprechen dem Zertifikatepreis. Je mehr Emittenten teilnehmen, je mehr Schadstoffe einbezogen werden etc., umso besser können die bestehenden Unterschiede in den Grenzvermeidungskosten ausgenutzt und ausgeglichen werden - umso effizienter arbeitet das System. Dieses Argument gilt übrigens auch für Ökosteuer-Regimes (hier pendeln sich die Grenzvermeidungskosten auf den Steuerbetrag statt auf den Zertifikatepreis ein).

- und weil ansonsten kein effizienter Handel zustande kommen würde (dieses Argument gilt nicht für Steuer-, sondern nur für Handelsregimes). Je enger die Märkte, umso höher sind die Preisausschläge. Die sich einstellenden Zertifikatpreise wären in engen Märkten mehr als alles andere vom Zufall bestimmt. Ein Unternehmer, der Vermeidungsanstrengungen unternimmt, könnte bei fortwährend und stark schwankenden Preisen nicht einschätzen, zu welchem Preis er die ,freien' Zertifikate in Zukunft veräußern kann. Die bestehende Unsicherheit hinsichtlich des Erfolgs der Vermeidungsanstrengungen würde dazu führen, dass die Emissionsvermeidung hinter dem theoretisch möglichen Niveau zurückbleibt.

Ein großes Format kann in räumlicher, zeitlicher, gegenständlicher und subjektiver Sicht angestrebt werden:

- In räumlicher Hinsicht wird in einem Cap-and-TradeSystem der ,Cap“ zur Limitierung der Inanspruchnahme des Umweltgutes idealerweise global gesetzt. D.h., im Idealfall werden in den Emissionshandel möglichst alle Länder einbezogen. Dies ergibt auch deswegen einen Sinn, weil es sich bei der Klimaproblematik um ein globales Problem handelt. So wurde und wird auch im Kyoto-Prozess die Integration vieler Länder angestrebt.

- Hinsichtlich der gegenständlichen Dimension kann ein möglichst großes Format erzielt werden, indem mehrere Schadstoffe zu einem ,Universalzertifikat' zusammengefasst werden. Anstatt vieler kleiner Märkte für die verschiedensten Treibhausgase entsteht so ein großer Markt für das Universalzertifikat. So wurden im Rahmen des Kyoto-Regimes (bislang: nur Handel zwischen
Staaten) verschiedene Treibhausgase in $\mathrm{CO}_{2}$-Äquivalente umgerechnet und dann eben dieses als Referenzobjekt (,Numeraire') handelbar gemacht. Auch in den europäischen Emissionshandel (Handel zwischen Unternehmen) sollen nach diesem Vorbild noch weitere Treibhausgase einbezogen werden.

- Die Effizienz des Marktes kann auch vergrößert werden, indem möglichst viele Anbieter und Nachfrager (Wirtschaftssubjekte) am Zertifikatehandel teilnehmen. Daher geht die Diskussion um die Einbeziehung weiterer Branchen in den Emissionshandel (v. a. Luftverkehr, wobei auch der Emissionsbeitrag des Luftverkehrs eine große Rolle spielt) sowie von NGOs und sogar von Privatpersonen.

- Das Emissionshandelssystem schöpft seine Effizienz aus dem Ausgleich von Grenzvermeidungskosten der verschiedenen Emittenten. Der Ausgleich der Unterschiede in den Grenzvermeidungskosten kann aber nicht nur zwischen den Emittenten, sondern auch auf der Zeitachse vorgenommen werden. Daher wird ein Ausschöpfen von Unterschieden in den Grenzvermeidungskosten über verschiedene Perioden diskutiert (,Banking' und ,Borrowing').

Wie alle marktwirtschaftlichen Instrumente des Umweltschutzes eröffnet also auch der Emissionshandel die Möglichkeit, Emissionen dort zu vermeiden, wo dies kostengünstig möglich ist und die Vermeidung dort zu unterlassen, wo dies teuer kommt. Das Cap-and-Trade-Regime macht es möglich, unter Beachtung des Umweltziels (Cap als maximale Umweltbelastung) die individuellen Überschüsse und in der Aggregation auch den sozialen Überschuss zu maximieren.

So gut und vernünftig diese Konzeption klingen mag sie hat einen entscheidenden Haken. Dieser besteht darin, dass das Umweltziel als ein Punktziel definiert wird: Der Zertifikatepreis bildet sich im Schnittpunkt der Kurve der aggregierten Grenzvermeidungskosten (Nachfrage nach Emissionszertifikaten) mit der Angebotskurve, resultierend aus einem möglichst universellen, großformatig definierten Cap (Angebot an Emissionszertifikaten). Damit wird eben auch das Umweltziel großformatig (im Klimakontext z.B. als globales $\mathrm{CO}_{2}$-Ziel) gesetzt. Die Systemlogik lautet dabei: Wo und wann die Vermeidung stattfindet oder aus Kostengründen eben nicht vermieden wird, ist sekundär; wichtig ist, dass das Ziel in der Summe (über die Räume, Spezies, Zeit und Schadstoffe hin) erreicht wird. Diese Logik impliziert, dass zeitliche, räumliche, gegenständliche oder auf Subjekte bezogene Emissionszusammenballungen und -spitzen in Kauf genommen werden. So können z. B. Hotspots auch bei bestimmten Klimagasen (so bei Ozon und $\mathrm{NO}_{\mathrm{x}}$ ) auftreten. Über Ballungsräumen mit einer hohen Emissionsdichte von Stickoxiden $\left(\mathrm{NO}_{\mathrm{x}}\right)$, Kohlenmonoxid $(\mathrm{CO})$ und flüchtigen organischen Verbindungen kommt es bei intensiver Son- 
neneinstrahlung (sommerliches Hochdruckwetter) immer häufiger zu relativ hohen Ozonbelastungen (Sommersmog). Eigentlich liegt es in der Effizienzlogik des Emissionshandelssystems, derartige Hotspots zu tolerieren. (Ordnungsrechtliche) Maßnahmen gegen Hotspots, wie sie faktisch angewendet werden, durchbrechen diese Logik und tragen damit Ineffizienzen in das System (s. unten).

Hingegen widerspricht die Rücksichtnahme auf die Resilienz der ökologischen Teilsysteme der Effizienzlogik, die ja u.a. den Verzicht auf Vermeidungsanstrengungen aus Kostengründen einfordert. Damit werden aber die Unterschiede bezüglich der Empfindlichkeiten der jeweiligen ökologischen Subsysteme (und damit die unterschiedlichen Grenzschadenskosten) im Grundsatz ignoriert. Wenn ein falsch gesetztes Umweltziel in effizienter Weise - eben durch ein großformatiges System, das auf Sensitivitäten keine Rücksicht nimmt - verfolgt wird, können aber Umweltbeeinträchtigungen die Folge sein. In der nachfolgenden Illustration dieser Aussage sei vereinfachend unterstellt, dass das theoretisch exakte Konzept der Identität der Kurven der aggregierten Grenzvermeidungs- und Grenzschadenskosten (,Internalisierungskonzept ${ }^{`}$ ) und die standardorientierte Politik (s. Abb. 2) zum selben Ergebnis führen. Das von den Planern/Naturwissenschaftlern gesetzte Belastungsmaximum entspricht also dem ökonomischen Optimalpunkt. Auf eine mathematische Beweisführung wird bei der nachfolgenden Kritik aus Platzgründen verzichtet. Stattdessen wird die Argumentation anhand Abb. 3 illustriert, wobei beispielhaft ein örtlicher Bezug (,Schadstoff' statt ,Ort', ,Zeit' oder ,Spezies` A bzw. B) gewählt werden soll: Die Grenzvermeidungskostenkurven sind $\mathrm{GVK}_{\mathrm{A}}$ für Gas $\mathrm{A}$ bzw. $\mathrm{GVK}_{\mathrm{B}}$ für

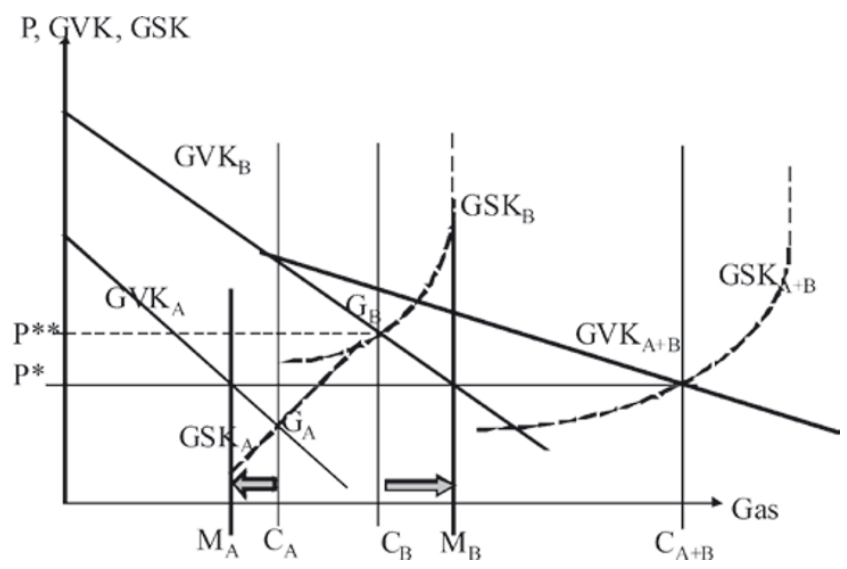

Abb. 3 Fehlsteuerungen bei einem Universalzertifikat. P Preis; GVK Grenzvermeidungskosten; GSK Grenzschadenskosten; C Belastungsmaximum nach naturwissenschaftlichen Maßgaben; $M$ die sich durch den Handel im Cap-and-Trade-System einstellende Menge (Die Abbildung ist notgedrungen simplifizierend; u. a. ist der Verlauf der Grenzvermeidungskostenkurven und der unterstellte lineare Zusammenhang zwischen Grenzvermeidungskosten und Ausbringungsmenge fragwürdig.)
Gas B. Die angesichts der (gestrichelt gezeichneten) Grenzschadenskosten (GSK) maximal tolerierbare Emissionsmenge für Gas $A$ sei $C_{A}$, diejenige für Gas $B$ sei $C_{B}$. Für die Grenzschadensfunktion von B ist ein nichtlinearer Verlauf angedeutet (kein Polynom ersten Grades). Der durch Gas B verursachte Grenzschaden (in der untenstehenden Graphik durch eine gestrichelte Linie gekennzeichnet) kann in Äquivalenten ( $\ddot{\mathrm{A}}$, analog zu den oben angesprochenen GWPs) des Grenzschadens ausgedrückt werden, der von Gas A verursacht wird: $\mathrm{GSK}_{\mathrm{B}}=\ddot{\mathrm{A}} \times \mathrm{GSK}_{\mathrm{A}}$. Derartige Äquivalente ermöglichen erst die Definition eines gesamthaften Cap und stellen daher in gegenständlicher Hinsicht eine conditio sine qua non für ein großformatiges Regime dar. Transformiert man also - im Sinne einer, geglückten' standardorientierten Politik - die naturwissenschaftlichen Erkenntnisse bezüglich der Äquivalente in entsprechende Grenzwerte (die dann zugleich Caps darstellen), muss sich $\mathrm{C}_{\mathrm{B}}=\ddot{\mathrm{A}} \times \mathrm{C}_{\mathrm{A}}$ ergeben. Werden nun die Grenzwerte von Gas A und $\mathrm{B}$ aggregiert, erhält man einen gesamthaften Grenzwert/Cap $\mathrm{C}_{\mathrm{A}+\mathrm{B}}=\mathrm{C}_{\mathrm{A}} \times \ddot{\mathrm{A}}$ $+\mathrm{C}_{\mathrm{A}}$. Problematisch an den Äquivalenten ist jedoch, dass sie nur eine Durchschnittsbetrachtung darstellen. Bezogen auf den Emissionshandel bedeutet dies: U. a. können regionale Spezifika (Luftdruck, Temperatur etc. ${ }^{3}$ ) lokal andere als die aggregierten Äquivalente verursachen. Hinzu kommen noch die jeweiligen Sensitivitäten und Expositionen der Schutzgüter und Ökosysteme vor dem Hintergrund unterschiedlicher Schadstoffkonzentration und -interaktionen.

Aus dem Schnittpunkt des aggregierten Cap (also: $\mathrm{X}^{*}=$ $\mathrm{C}_{\mathrm{A}+\mathrm{B}}=\mathrm{C}_{\mathrm{A}} \times \ddot{\mathrm{A}}+\mathrm{C}_{\mathrm{A}}$ ) sowie der aggregierten Grenzvermeidungskostenkurve ergibt sich nun der Einheitspreis für die Universalzertifikate P*. Der Schnittpunkt zwischen den Grenzvermeidungskosten und den Grenzschadenskosten für A und B bezeichnet jeweils die lokalen Teiloptima. Der Einheitspreis P* führt aber dazu, dass im Regelfall das Gesamtoptimum nicht auf die - an den jeweiligen Teiloptima orientierten - Grenzwerte/Caps $\mathrm{C}_{\mathrm{A}}$ und $\mathrm{C}_{\mathrm{B}}$, heruntergebrochen' werden kann. Für den Emittenten spielen ja die jeweiligen Caps $C_{A}$ und $C_{B}$ keine Rolle; lediglich der aggregierte Cap $\mathrm{C}_{\mathrm{A}+\mathrm{B}}$ wird in ein Preissignal transformiert. Der jeweilige Emittent orientiert sich damit nur an den individuellen Grenzvermeidungskosten und dem Preis P*. Demnach stellt sich für jedes Gas im Schnittpunkt zwischen Grenzvermeidungskostenkurve $\left(G V K_{A}\right.$ bzw. $\left.G_{V K}\right)$ und $P^{*}$ eine Menge $\left(\mathrm{M}_{\mathrm{A}}\right.$ bzw. $\left.\mathrm{M}_{\mathrm{B}}\right)$ ein, die vom lokalen Cap $\left(\mathrm{C}_{\mathrm{A}}\right.$ bzw. $\left.\mathrm{C}_{\mathrm{B}}\right)$ regelmäßig mehr oder weniger abweicht. Dies bedeutet: Von Gas A wird weniger, von Gas B mehr emittiert, als dies angesichts der Exposition und Sensitivität der jeweiligen Schutzgüter in der Region nach naturwissenschaftlicher Maßgabe vertretbar ist (differenzierte Grenzwertsetzungen würden sich an $\mathrm{C}_{\mathrm{A}}$ und $\mathrm{C}_{\mathrm{B}}$ orientieren). Will man das Regime nicht

\footnotetext{
${ }^{3}$ Diese Faktoren sind maßgeblich für die Diffusionseigenschaften eines Schadstoffes verantwortlich.
} 
durch eine Regulierung in seiner Effizienz beschränken, muss diese Zielverfehlung hingenommen werden.

Gegen diese Darstellung wird häufig eingewendet, dass speziell bei $\mathrm{CO}_{2}$ die Strukturproblematik nicht bestehe; hier sei vielmehr eine globale Betrachtung angemessen. Dieser Einwand trifft jedoch nicht $\mathrm{zu}$, was anhand des folgenden Beispiels gezeigt werden soll. Dabei wollen wir nach Orten anstatt nach Gasen differenzieren: Es sei A (stellvertretend für andere Ökosysteme) das Alpenvorland und B ein großes Vorkommen von riffbildenden Kaltwasserkorallen, z. B. vor der Küste Norwegens. ${ }^{4}$ Das Korallenriff geht zusammen mit seinen Bewohnern wegen der $\mathrm{CO}_{2}$-bedingten - rasch voranschreitenden - Übersauerung des Meeres zugrunde, was zu einem weiteren Verlust an Biodiversität ${ }^{5}$ beiträgt; das Alpenvorland mag hingegen in seiner Biodiversität durch die steigende $\mathrm{CO}_{2}$-Konzentration weitgehend unberührt bleiben.

Nun ist die Unwissenheit über das Funktionieren der Ökosysteme groß, und zwar umso größer, je weiter die Systemabgrenzung gewählt wird. Über die Rückkopplungseffekte auf andere Ökosysteme, die von den sterbenden Korallen ausgehen, können daher nur Vermutungen angestellt werden. Das Überschreiten der Resilienzen der Ozeane aufgrund weiterer $\mathrm{CO}_{2}$-Einträge (Produktion von Kohlensäure, $\mathrm{H}_{2} \mathrm{CO}_{3}$ ) wird jedenfalls für möglich gehalten. ${ }^{6}$ Hinsichtlich des Korallenriffs besteht diesbezüglich im Beispiel das Problem einer möglichen nichtlinearen lokalen Grenzschadensfunktion: Selbst wenn der Cap gesamthaft eingehalten wird, findet doch lokal eine Überschreitung der Schwellenwerte statt, so dass die Resilienz des Subsystems Korallenriff überstrapaziert wird. In $\mathrm{M}_{\mathrm{B}}$ geht die Grenzschadensfunktion gegen unendlich. Die Folge: Möglicherweise kippt nicht nur das Subsystem Korallenriff, sondern wegen der Rückwirkungen auf A sogar das gesamte System (im Meer, und im Gefolge davon abhängige Systeme auf dem Land). ${ }^{7}$ Es ergibt sich folgendes Bild: Primär effizienzorientierten Regi-

\footnotetext{
${ }^{4}$ Diese ,Lophelia pertusa' sind tatsächlich riffbildend. http://www. co2-handel.de/article311_2945.html [16.8.2008]

${ }^{5}$ In Artikel 2 der Konvention über biologische Vielfalt der Vereinten Nationen wird Biodiversität definiert als ,... the variability among living organisms from all sources including, inter alia, terrestrial, marine and other aquatic ecosystems, and the ecological complexes of which they are part; this includes diversity within species, between species and of ecosystems." (Secretary of the Convention on Biological Diversity 2001).

${ }^{6}$ Die Kohlensäure zerfällt in verschiedene Ionen, nämlich zunächst in Bikarbonat $\left(\mathrm{HCO}_{3}^{-}\right)$und weiter in Karbonat $\left(\mathrm{CO}_{32}-\right)$, wobei ein bzw. zwei Wasserstoffionen $(\mathrm{H}+)$ freiwerden. Diese Ionen bestimmen den $\mathrm{pH}$-Wert, je höher ihre Konzentration, umso niedriger der $\mathrm{pH}$, umso saurer die Lösung. Steigt die $\mathrm{CO}_{2}$-Konzentration im Wasser, da mehr Kohlendioxid aus der Atmosphäre aufgenommen wird, entsteht mehr Bikarbonat und so mehr H+-Ionen - Vgl. http://www.scinexx.de/wissen-aktuell-7294-2007-10-25.html [16.8.2008]

${ }^{7}$ Dies ist natürlich ein hypothetisches Horrorszenario zu Illustrationszwecken.
}

mes ermangelt es an Rücksichtnahme auf die Resilienz von Ökosystemen; gleichzeitig bestehen große Wissenslücken bezüglich der Funktionsweise von (Öko-)Systemen und ihrer Störanfälligkeit: Ökologische Systeme haben einen nicht linearen Charakter, es existieren zeitverzögerte Wirkungen aufgrund von Pufferungen sowie Synergieeffekte; die Komplexität vernetzter Regelkreise ist weitgehend undurchschaubar. Angesichts unserer Unwissenheit über diese Aspekte und vor dem Hintergrund der Bedeutung der Lebens- und Entwicklungsfähigkeit von Ökosystemen für das menschliche Überleben darf diese nicht dem Streben nach Effizienz nachgeordnet werden. Grundsätzliche Bedenken bestehen ferner dahingehend, die verschiedenen Leitwertdimensionen (Effizienz, Sicherheit etc.) eines Systems in eine einzige Dimension - nämlich Geld - zu aggregieren, wie dies durch den Zertifikatepreis im Cap-and-Trade-Regime geschieht. Der letztlich die verschiedenen Systemdimensionen zusammenfassende Preis nährt die Illusion, es gäbe ein eindeutiges, konfliktfreies Kalkül einer ökonomischen Effizienz (Optimum). Mehrdimensionalität führt hingegen zu (produktiven) Konflikten, die sich oftmals ökonomischen Kategorien entziehen und (politischer) Kompromisse bedürfen.

Das Gesamtsystem (hier: Meer) kann nur geschützt werden, indem außerökonomisch bestimmte Grenzen errichtet und respektiert werden; in der Terminologie Ciriacy-Wantrups (1968) ist der ,Safe Minimum Standard“ zu beachten. Wo die betreffenden Grenzen liegen, ist jedoch oft ungewiss. Mit der Ungewissheit über Systemzusammenhänge sollte in rationaler Weise jedoch nach dem ,Vorsorgeprinzip“ (vgl. Gruhl 1976; Costanza et al. 2001) umgegangen werden. Demnach sollte nicht auf sichere wissenschaftliche Erkenntnisse gewartet, sondern die potenziellen Umweltschäden durch (behördliches) Handeln antizipiert werden. Die Abwesenheit eines Beweises, wo der Umschlagspunkt für das jeweilige ökologische Teilsystem liegt, bedeutet nämlich nicht den Beweis der Abwesenheit derartiger Umschlagspunkte (Wuppertal Institut für Klima, Umwelt und Energie 2005; Meyerhoff 1999). Selbstverständlich liefern die nach außerökonomischen Maßstäben gesetzten Grenzen keine Garantie für ein perfektes, über alle Zweifel erhabenes Kalkül. ,Aber das ist kein Nachteil, denn nicht eine unvollkommene, immer wieder Diskussion und Nachdenken erfordernde Strategie ist problematisch, sondern die Illusion einer perfekten Strategie, der alle folgen, ohne Verantwortung übernehmen zu müssen. "8

Die Orientierung am Safe Minimum Standard würde vorliegend jedoch nicht den sich im Zuge des Emissionshandels einstellenden Preis P* (der eben die Durchschnittsbetrachtung wiedergibt), sondern einen höheren Preis $\mathrm{P}^{* *}$

${ }^{8}$ E-Mail von K.-F. Müller-Reißmann vom 25.7.2008 an den Verfasser. 
erfordern, der Rücksicht auf das ,schwächste Glied der Kette' nimmt (nämlich das Korallenriff). Anders formuliert: Von den Marktteilnehmern wäre nach dem Vorsichtsprinzip in Gestalt dieses Aufpreises eine Prämie für die Erhaltung einer wichtigen Option für die Nachwelt einzufordern. Die Folge wären jedoch „zu hohe“ Vermeidungsanstrengungen in Ort A. Auch dies illustriert den Zielkonflikt zwischen Effizienz und Systemsicherheit (als weiteren Leitwert). Aus dem Effizienzkriterium lässt sich eben kein Optimum ableiten, bei dem alles ,automatisch', wie durch eine ,unsichtbare Hand' bestens geregelt wäre.

Die dargestellten Probleme könnten theoretisch durch ausdifferenzierte Märkte für A und B gelöst werden. Dann könnten sich für jedes Marktsegment eigenständige, zielführende Preise bilden. Allerdings wäre ein derartiges, kleinteiliges Design nicht mehr effizient (vgl. die Ausführungen oben): Je enger die Märkte definiert werden, umso geringer das Potenzial an auszugleichenden Grenzvermeidungskosten, und damit die Effizienz. Es bestehen zudem Zweifel, ob die entstehenden, mehr oder weniger engen Märkte zu einer effizienten Preisbildung führen würden (Volatilität der Preise). Dies gilt in gegenständlicher Hinsicht z. B. für die in Kyoto einbezogenen Gase $\mathrm{NO}_{\mathrm{x}}$ und $\mathrm{SF}_{6}$, und zwar selbst bei einem weltweiten Handel. Die Bedenken bleiben auch dann bestehen, wenn man in den engen Märkten Kurspflege über ,Emissions-Zentralbanken` o. dgl. betreiben würde.

Grundsätzlich gilt also: Es besteht ein Zielkonflikt zwischen der ökologischen Treffsicherheit (die enge Märkte erfordert) und der ökonomischen Effizienz (die man mit einem weiten Systemdesign erreicht). Entscheidet man sich - dem Dominanzanspruch der Effizienz folgend - für ein großformatiges Design, sind wegen der mangelnden Rücksichtnahme auf die Belastungsstrukturen und damit auf die Resilienzen der (Öko-)Systeme Reparatur- und Korrekturmechanismen nötig: Weil die unter Berücksichtigung der Resilienz der Ökosysteme oftmals erforderliche Steuerung einer Belastungsstruktur durch effizienzorientierte, marktwirtschaftliche Instrumente eben nicht geleistet werden kann, ${ }^{9}$ müssen zeitliche, räumliche, schadstoff- und/oder personenbezogene Belastungsspitzen (,Hotspots') durch andere Mechanismen vermieden werden. Auf der Hand liegen dabei ordnungsrechtliche Vorgaben. So wird z. B. in der IVU-Richtlinie (1996) den nationalen Umweltbehörden ein Einschreiten gestattet, wenn eine, erhebliche lokale Umweltverschmutzung' droht. Damit wird zwar das oben schon angesprochene Problem bezüglich Hotspots (Sommersmog etc.) entschärft; allerdings wird dem Emissionshandel dann auch seine Effizienz genommen. Dasselbe trifft auch zu, wenn produktionstechnisch verbundene Schadstoffe in unterschiedlichen Regimes geregelt sind: Wird z. B. einerseits

\footnotetext{
${ }^{9} \mathrm{Zu}$ den diesbezüglichen Problemen mit Blick auf den Luftverkehr
} vgl. Scheelhaase und Grimme (2006). ein Schadstoff im Emissionshandel einem Cap unterzogen und unterliegt ein mit diesem im Wege der Kuppelproduktion verbundener Schadstoff (wegen seiner Gefährlichkeit oder schnellen Diffusion, wie z.B. $\mathrm{O}_{3}-$ mit einem rund 2000-fachen Treibhauspotenzial, verglichen mit $\mathrm{CO}_{2}$ ) dem Ordnungsrecht, so werden auch bezüglich dem im Emissionshandel unterworfenen Schadstoff mittelbar Grenzwerte gesetzt. Auch diese ,Reparaturmaßnahme“ hätte wie sämtliche Korrekturen negative Rückwirkungen auf die Effizienz des Regimes und widerspricht so der immanenten Logik des Handelsregimes. Die ordnungsrechtlichen Korrekturen untergraben damit auch die klassische Rechtfertigung des Systems, die den Zielkonflikt zwischen Effizienz und ökologischer Treffsicherheit negiert. Insoweit sind die ordnungsrechtlichen Korrekturen keinesfalls eine neutrale ,Ergänzung', wie das manchmal dargestellt wird.

\subsection{Exkurs: Unsicherheit, Adaptivität, Wandlungsfähigkeit und Effizienz}

Unser Wissen über ökologische Systeme ist rudimentär, die diesbezüglichen Erkenntnisse unsicher. Die Zusammenhänge werden erst langsam und sukzessive aufgeklärt, manchmal kommt es zu Revisionen oder veränderten Sichtweisen. So ist teilweise noch nicht einmal die Zusammensetzung bestimmter schädigender Emissionen hinreichend klar (beispielsweise ist im Hinblick auf den - noch nicht in die Emissionshandelsregimes einbezogenen - Verkehrssektor die chemische Natur von 85 bis $90 \%$ der organisch-chemischen Emissionen von Kfz-Abgasen unbekannt, vgl. Wiedmann et al. 2000). Im - bis dato noch nicht in den Kyoto-Prozess einbezogenen - Luftverkehr geht ein erheblicher Anteil der Klimaschadenswirkungen von anderen Bestandteilen im Abgas als dem Kohlendioxid aus. Die Wirkungen von u. a. Stickoxiden, Kondensstreifen oder Zirruswolken sind ebenso wie die atmosphärischen Vorgänge in der Reiseflughöhe von $10 \mathrm{~km}$ nur teilweise erforscht (Wuppertal Institut für Klima, Umwelt und Energie 2005). Von einem effektiven Umweltschutzregime ist aber zu fordern, dass wissenschaftliche Erkenntnisfortschritte rasch und friktionslos integriert werden können. Andererseits kann es aber nicht im Interesse der Effizienz des Systems sein, wenn bei Vorliegen neuer wissenschaftlicher Erkenntnisse mit den Äquivalenten (oben: GWPs) auch fortwährend die Rahmenbedingungen des Regimes geändert werden. Hiermit würde eine neue Dimension von Systemunsicherheit eingeführt. Das Ergebnis wäre eine entsprechend hohe Volatilität der Preise und eine Beeinträchtigung des Vertrauens in den finanziellen Erfolg von Vermeidungsanstrengungen. Andererseits wäre - wenigstens bei global wirkenden Schadstoffen - genau diese Änderbarkeit des Systems eine wesentliche Voraussetzung für ein - nach naturwissenschaftlichen Maßstäben - korrektes Wirken. Die Möglichkeiten der Integration neuer 
Erkenntnisse in ein Cap-and-Trade-Regime sind also beschränkt; auch die Adaptivität und Wandlungsfähigkeit wird dem Effizienzkritierium untergeordnet.

\subsection{Zur Bedeutung von Effizienz und Vielfalt in lebenden Systemen}

Nachfolgend wird die Rolle von Effizienz in selbstorganisierenden (,lebenden') Systemen (Lebewesen, Unternehmen, Stadt, Gesellschaft, etc.) beleuchtet und mit der Bedeutung in umweltökonomischen Instrumenten als ,instrumentelle' oder ,Mittelsysteme' (wie z. B. technische Systeme) konfrontiert. ${ }^{10}$ Der Effizienzbegriff sagt etwas über das Verhältnis von Nutzen und Kosten (auch interpretierbar als negativer Nutzen) aus. Der Nutzen - und damit der Effizienzbegriff - bezieht sich aber immer auf einen bestimmten Zweck, ist also intentional. Mit Bezug auf ökologische Systeme kann man diesen (System-)Zweck insbesondere dann, wenn man die Systeme weit definiert, als die Sicherung des weiteren Überlebens und die weitere Entwicklung bezeichnen. Insbesondere selbstorganisierende, lebende Systeme (also auch Ökosysteme!) sind selbstreferentiell (Müller-Reißmann et al. 1988). ${ }^{11}$ Sie tragen ihren Zweck in sich selbst und sind nicht durch ihre Funktion für andere definiert. In lebenden Systemen ist, von Natur aus' immer schon eine gewisse Ineffizienz angelegt, zumal sie nicht gegen das Minimum streben, das durch das Gesetz der minimalen Entropieerzeugung beschrieben wird. Erst ab einer gewissen Entfernung von dieser Schwelle kann nämlich überhaupt ein offenes System, wie es Leben darstellt, entstehen (Prigonine und Stengers 1986, zit. in Müller-Reißmann et al. 1988). Dennoch benötigt jedes funktionierende lebende System ein Minimum an Effizienz. Effizienz ist die Systemantwort auf Ressourcenknappheit; hierbei handelt es sich aber um ein ubiquitäres Phänomen. Allgemein kann sich Effizienz auf Systemebene am besten in einer sicheren, strukturierten Umgebung entfalten, bei der relativ wenig Handlungsfreiheit, Wandlungsfähigkeit und Adaptivität notwendig ist. Je unsicherer jedoch die Umgebung, in der sich ein System befindet, umso mehr gewinnen - auf Kosten der Effizienz - die Vorkehrungen gegen die hieraus erwachsenden Bedrohungen und zur Nutzung der hieraus erwachsenden Chancen an Bedeutung. In einer ökonomischen Interpretation ist die beste Strategie gegen allfällige Unsi-

\footnotetext{
${ }^{10}$ Streng genommen ist es nicht ganz richtig, von Ökosystemen als ,lebenden Systemen' zu sprechen. Vielmehr handelt es sich um hochkomplexe, vielfältig vernetzte, in Konkurrenz und Kooperation stehende unterschiedliche, lebende Systeme'. Wenn Ökosysteme und ,lebende Systeme' nachfolgend nicht streng unterschieden werden, so deswegen, weil die Unterscheidung mit Blick auf das Ziel der Abhandlung nicht relevant ist.

${ }^{11}$ Varela et al. (1974) verwenden in diesem Kontext den Begriff der ,Autopoeisie“ (als den Prozess der Selbsterschaffung und -erhaltung eines Systems).
}

cherheiten die Schaffung von Optionen. Dies geschieht bei den Ökosystemen v. a. mit der ,Investition“ in Biodiversität. Vielfalt , an sich' (!) ist also von elementarer Bedeutung für das Überleben eines (Öko-)Systems in einer unsicheren Welt. Allerdings: Jeder Aufbau von Optionen als Strategie gegen die allfällige Unsicherheit (und hier: für die Fortentwicklung der Evolution) ist ,teuer'; eine solche ,Strategie bindet Ressourcen. Die Ineffizienzen des Systems nehmen daher vor allem mit der produzierten Vielfalt, also der Biodiversität, zu - wenngleich Effizienz und Vielfalt sich auch gegenseitig bis zu einem gewissen Grad bedingen. ${ }^{12}$ Das latente Spannungsverhältnis zwischen Effizienz und Vielfalt zeigt sich beispielsweise darin, dass von allen Lebensräumen auf dem Kontinent keineswegs der Tropenwald (mit seiner hohen Artenvielfalt) die höchste Effizienz aufweist, wenn man diese an der Sekundärproduktion misst (also die Fähigkeit, Tiere zu ernähren): Dies ist vielmehr die - relativ eintönige - Savanne. ${ }^{13}$

Die Ineffizienz, die mit der Vielfalt in ökologischen Systemen einhergeht, zeigt sich regelmäßig in Redundanzen (z.B. Funktionsdopplungen mit Blick auf das System). ${ }^{14}$ Hiermit geht jedoch ein ,Versicherungseffekt ${ }^{`}$ einher: Mit zunehmender Artenvielfalt nimmt die Wahrscheinlichkeit zu, dass zumindest einige Arten oder Genotypen in der Lage sind, funktionell unterschiedlich auf sich laufend ändernde Umweltzustände (endogener und v.a. exogener Art, z.B. aufgrund von Klimaschwankungen) zu reagieren und sich anzupassen (Adaptivität, Wandlungsfähigkeit). Ein Beispiel: Generalisten wie Spezialisten koexistieren in Ökosystemen bei partieller Funktionsüberschneidung hinsichtlich des Systems. Spezialisten nutzen dabei die Vorteile, die sie durch ihre Festlegungen haben. Sie sind auf feste Strukturen angewiesen und können durch ihre Festlegungen und Spezialisierungen eine relativ hohe Effizienz entwickeln. Dies ist eine der ,teuren', eigentlich ineffizienten Schaffung von Optionen diametral entgegengesetzte Strategie (SCNAT

\footnotetext{
${ }^{12}$ Man denke an das Ausmaß an Biomasseproduktion, das stark von der Biodiversität abhängt.

${ }^{13}$ Gehölze produzieren nämlich nur wenig photosynthetisch aktives Gewebe, der überwiegende Teil hat Stütz- oder Speicherfunktion. Gräser aber bestehen fast ausschließlich aus grünen Organen. In der Savanne leben auf einem ha $150-200 \mathrm{~kg}$ Tiere, während in einem ha Wald nur etwa $10 \mathrm{~kg}$ Tiere leben. Der Grund dafür liegt darin, dass Gras von den Tieren viel leichter aufgenommen und verwertet werden kann als Holz. Wegen der hohen Produktivität der Savanne wird diese vom Menschen als Weidegebiet für ihre Herden genutzt und auch häufig Waldgebiete in Grasland (Wiese, Weide oder Getreidefelder) umgebildet. Gefährlich werden diese Handlungen derzeit vor allem dem tropischen Regenwald, der nach und nach gerodet wird, um in kurzzeitig nutzbares Ackerland umgebildet zu werden. Die Savanne mit ihrem Effizienzmaximum steht zwischen den Wüsten (geringe Produktivität) und dem tropischen Regenwald - vgl. http://www.myss.de/science/ oekologie/oekosysteme.html.

${ }^{14}$ Hierzu und zu anderen Aspekten wie dem ,Overyieldingeffekt ${ }^{\star}$ und dem ,Kovarianzeffekt‘ vgl. Baumgärtner und Schiller 2001.
} 
2008). Andererseits zeigt sich bei Änderungen in den Umweltbedingungen, wie die Festlegungen der Spezialisten zum Risiko mutieren können: Angesichts sich ändernder Umweltbedingungen haben wohl die eigentlich ineffizienteren Generalisten die höheren Überlebenschancen (o.V. 2001). Dann stellen sich auch die an sich ineffizienten partiellen ,Funktionsüberschneidungen“ von Generalisten und Spezialisten plötzlich als Vorteil heraus. Nur dann, wenn die Systemfunktion der verschwindenden Art durch andere Arten übernommen werden kann, bedroht das Aussterben der Art das System nicht als Ganzes. Nur vor diesem Hintergrund kann sich das System die unterlassene ,Investition in Versicherungsmaßnahmen' durch die spezialisierten Arten auch, leisten'.

Es zeigt sich also: Effizienz ist insbesondere in solchen lebendigen Systemen, die mit einer unsicheren Umgebung konfrontiert sind, eher auf der Ebene der einzelnen Systemelemente, weniger auf der Ebene des Gesamtsystems von Bedeutung. Die einzelnen Systemelemente folgen insoweit partiell einer anderen Logik als das Systemganze. Im Gegensatz zu der relativen Rolle der Effizienz in natürlichen Systemen als Ganzes wird das Effizienzpostulat von der (Umwelt-)Ökonomie absolut gesetzt. Effizienz wird für die einzelnen Elemente des Mittelsystems (Gas oder Ort A bzw. B im vorigen Abschnitt), und das Gesamtsystem gleichermaßen eingefordert. Dies liegt ganz in der Aggregationslogik der herkömmlichen Ökonomie, der zufolge das Ganze die Summe seiner Teile ist (Keen 2004). Dagegen steht die Erkenntnis der Systemforschung: „Systemverhalten lässt sich durch die Eigenschaften der Komponenten allein nicht erklären; das System ist mehr als die Summe seiner Teile. Systemverhalten ist mehr als die Summe seiner Teile. Systemverhalten ist daher eine neu auftauchende emergente Eigenschaft des Systems, das heißt Resultat seiner besonderen Konfiguration von Systemelementen und ihrer Verbindungsstruktur." (Bossel 1998).

Auch für ein erfolgreiches umweltökonomisches ,Mittelsystem ' gilt die Forderung, dass sich die besonderen Merkmale seiner Umwelt in seiner Struktur und seinen Funktionen wiederspiegeln sollen (Bossel 1998). Mit der Absolutsetzung und Überbetonung des Leitwertes der Effizienz bestehen hier aber v.a. auf der Ebene des Gesamtsystems große Zweifel an einer Vereinbarkeit mit den Funktionsnotwendigkeiten der ökologischen Systeme. Stellt man sich auf den Standpunkt, dass das Systemganze regelmäßig einer anderen Logik folgt als die Systemteile, muss man zu dem Schluss kommen, dass mit den gängigen, auf Effizienz fokussierten ökonomischen Instrumenten des Umweltschutzes den Ökosystemen eine fremde Logik übergestülpt wird. Vor diesem Hintergrund erscheint ein als absolut gesetztes Effizienzziel als eine Ausdrucksform des ,Imperialismus', welcher der zeitgenössischen Ökonomie im Sinne einer Verallgemeinerung der ökonomischen Prinzipien auf alle Lebenszusammenhänge oft vorgeworfen wird. ${ }^{15}$

\section{Zeiteffizienz und Resilienz - die Rolle von Rentabilität und Zins}

\subsection{Zeit ist Geld}

Nachfolgend geht es um Zeiteffizienz, also das Bestreben, ein bestimmtes Ziel mit einem möglichst geringen zeitlichen Aufwand zu erreichen. Das Konzept der Zeiteffizienz ist schon im utilitaristischen Nutzenkalkül angelegt, das der herrschenden ökonomischen Lehre zugrunde liegt: Dabei geht es darum, den Barwert der Differenz aus Nutzen und Kosten (Nettonutzen) zu maximieren (zur Kritik vgl. Löhr 2009). Das Barwertkalkül hängt mit den Renditeforderungen der Anleger bzw. dem positiven Zins zusammen: ${ }^{16}$ Ein Euro, der heute eingenommen wird, ist bei einem positiven Zinssatz nämlich mehr wert als ein Euro, der erst in einem Jahr in die Kasse fließt. In der Zwischenzeit hätte der Euro nämlich zinstragend angelegt werden können. Je später das Geld zufließt, umso höher sind daher die entgangenen Zinsen (Opportunitätskosten). Umgekehrt können mit einem Euro noch umso länger (und mehr) Zinserträge erwirtschaftet werden, je später er ausgegeben wird. Das Ziel, auch über die Zeitschiene hinweg den Nutzen zu maximieren, wird also bei einem positiven Zinssatz dadurch erreicht, dass die Nutzen des Wirtschaftens möglichst in die Gegenwart gezogen und die Kosten in die Zukunft, eventuell auf künftige Generationen verschoben werden. Je höher der Zins bzw. die Renditeforderungen der Anleger, umso stärker ist dieser Anreiz. Die Konsequenzen sind mannigfaltig. Hier nur wenige Beispiele: Regenerative Energieträger zeichnen sich häufig gerade dadurch aus, dass sie - im Vergleich zu ihrer fossilen Konkurrenz - hohe Anfangsauszahlungen aufweisen. Die vergleichsweise teurere Erstinvestition wird im Barwertkalkül hoch gewichtet. Die durch die größere Ressourceneffizienz entstehenden Ersparnisse fallen hingegen erst in der Zukunft an, gehen also mit einem geringeren Gewicht in das Barwertkalkül ein. Daher sind die betreffenden Energien häufig nicht ohne weitergehende Maßnahmen (wie Subventionen) wettbewerbsfähig. Mit anderen Worten: Die technisch denkbare Vielfalt der möglichen Energieträger wird durch die Renditehürde beschnit-

\footnotetext{
${ }^{15}$ In diesem Sinne ist die herrschende Ökonomie auch im Sinne von Emile Durkheim reduktionistisch, wenn man unter Reduktionismus die Erklärung von Phänomenen auf einer ,höheren Ebene‘ (z. B. das soziale Verhalten von Menschen) allein durch Gesetzmäßigkeiten einer ,niederen“ Ebene (z. B. der Chemie) versteht. Vgl. die Beiträge in Henkel et al. 1998.

${ }^{16}$ Darüber, ob die Zinsforderungen in den Präferenzen der Menschen oder nicht vielmehr im Geld selber verankert sind, vgl. Löhr 2009.
} 
ten. Analoges passiert auf anderen, benachbarten Gebieten: Beispielsweise ist die Kultivierung einer nachwachsenden Ressource (z. B. ,Bioenergie'), die mit einer höheren Rate als der Zinssatz wächst, ökonomisch effizient - wenngleich in der Konsequenz Monokulturen entstehen (s. das Beispiel der Eukalyptusbäume, vgl. Costanza et al. 2001). Wieder verhindert die Renditehürde eine größere Vielfalt.

Erst über den Zins wird Zeit und Geld synonym gemacht. Das Rentabilitätsprinzip fordert ein, keine Zeit zu vertrödeln. Weil beispielsweise Vorratslager Zinsen kosten, werden diese auf die Autobahn gelegt (,Just-in-time-Produktion'), welche die Landschaft zerschneidet sowie neue Stoffströme und Emissionen generiert. Andere Verkehrsmittel, die weniger schnell sind, werden uninteressant - die Vielfalt an Formen wird erneut reduziert.

Zeiteffizienz kann durchaus in Gegensatz zur Ressourceneffizienz stehen. Je höher die Renditeforderungen, umso wichtiger wird die möglichst rasche Generierung von (monetären) Nutzen - dies gilt selbst dann, wenn hierbei entsprechend höhere Kosten (Ressourcenverbrauch) entstehen, wenn diese nur in die Zukunft verschoben und damit abgezinst, also geringer gewichtet werden können. Zeiteffizienz erfordert, dass Mensch oder Material mit geringstem Zeitaufwand von Punkt A nach B gelangen kann. Das Mittel hierzu ist häufig die Begradigung der ,Meander der Stoffströme'. Hierbei geht es um wesentlich mehr als das sich unwillkürlich aufdrängende Beispiel einer Flussbegradigung. Ein wichtiges Beispiel ist eben auch die Nutzung fossiler Energien, auf die sich weite Teile unserer Wirtschaftstätigkeit stützen. Kunstler (2005) betont, dass die Natur in ihren Systemen ,Hindernisse' eingebaut hat, welche die Energieflüsse in einem ,Check and balance' verlangsamen und damit die Leistungsfähigkeit begrenzen, zugleich aber auch die Steigerung der Entropie (unbrauchbare Abwärme) ihrer Systeme bremsen und diese oft sehr komplex erscheinen lassen: "The built-in constraints of inefficient biogenic economies reduce the flow of the potential, often to the point where systems based on inefficient economies last for geological epochs, not just a few decades in the case of a fish hatchery. Everything that we identify with nature takes the form of inefficient systems." Kunstler begreift andererseits die gesamte moderne, auf der Nutzung weniger Energieträger wie Öl, Erdgas, Kohle und Uran basierende Technologie (auf der unsere Lebensweise, zumindest in der Ersten Welt, zum allergrößten Teil beruht) als ein ,Niederreißen dieser Barrieren, als eine ,Begradigung der Meander ${ }^{6}$ der Stoffströme mit dem Ziel, die (Zeit-)Effizienz noch weiter zu erhöhen. Bei einer einseitigen Ausrichtung am Kriterium der Zeiteffizienz gilt: „Efficiency is the straightest path to hell." (Kunstler 2005). Die Rücksicht auf andere (z. B. soziale und ökologische Systeme erfordert jedoch den Respekt vor Schranken und außerökonomischen Zielen: „There have to be limits." (Kunstler 2005).
3.2 Das Rentabilitätsprinzip als chrematistische Perversion

(Zeit-)Effizienz, das haben wir oben schon betont, ist ein intentionaler Begriff. Er bezieht sich also auf einen bestimmten (System-)Zweck. Schenkt man den volkswirtschaftlichen Lehrbüchern Glauben, ist der Zweck des Wirtschaftens der möglichst präferenzengerechte Konsum. Die Sphären von Investition und Ersparnis, in der Gelder zinstragend angelegt werden, sind demnach nur Mittel, um diesen Zweck zu erreichen. Aus Gründen, die ich mangels Raum hier nicht weiter erörtern kann, aber an anderer Stelle ausführlich beschrieben habe (Löhr 2009), verhält es sich in der kapitalistischen Realität genau umgekehrt: Zweck und Mittel des Wirtschaftens werden pervertiert. Zweck ist die Erzielung von Rendite. Der Konsum wird zum Mittel degradiert, um eben den Zweck der Befriedigung der Anlegerinteressen zu erreichen. Die Sphäre von Investition und Ersparnis verselbstständigt sich. Shareholder Value wird zum Maßstab aller Dinge. Diese Verkehrung von Mittel und Zweck, die Fokussierung auf die Anlegerinteressen möchte ich ,Rentabilitätsprinzip" nennen. Schon Aristoteles unterschied zwischen der ,natürlichen Erwerbskunst ${ }^{\star}$, also der Produktion für den Bedarf (,Ökonomik') und der ,widernatürlichen' Erwerbskunst, der Produktion für Handel und Gewinn und Akkumulation von Geld (,Chrematistik ${ }^{617}$ ).

Aus dieser Pervertierung von Mittel und Zweck ergibt sich auch die eigenartige Intentionalität des auf das Rentabilitätsprinzip gerichteten Wirtschaftens: Diese ist dahingehend reduziert, dass unabhängig von anderen wesentlichen Aspekten wie dem Bedarf, ja sogar unabhängig von der Wirtschaftlichkeit die Daseinsberechtigung allen Projekten versagt wird, die nicht die geforderte Rendite erwirtschaften. Beispielsweise kann ein Projekt durchaus ,schwarze Zahlen schreiben'; wenn die Rendite aber unterhalb der geforderten Verzinsung der Kapitalgeber liegt, bedeutet dies das ,Aus‘. Über das Rentabilitätsprinzip wird die Effizienz von einem ,Leitwert‘ (Bossel 1998) unter anderen zum Selbstzweck des kapitalistischen Wirtschaftssystems überhöht. Selbst, wenn es - entgegen den obigen Ausführungen - möglich wäre, ein ökologisch treffsicheres Cap-and-Trade-Regime zu implementieren: Solange die ,chrematistische Perversion' des Rentabilitätsprinzips bestehen bleibt, wäre dieser Erfolg nur sehr beschränkt.

\section{Ein Plädoyer für die Neubewertung des Effizienzkriteriums}

Nach der systemaren Leitwerttheorie stehen alle Leitwerte eines Systems in einem latenten Spannungsverhältnis zuei-

\footnotetext{
${ }^{17}$ Aristoteles stellt die Chrematistik der Ökonomik gegenüber. Bei der Chrematistik geht es nicht um die Deckung von Bedarf, sondern um die Akkumulation von Geld (vgl. Hesse 2003).
} 
nander. Die Überbetonung oder Vernachlässigung einzelner Leitwerte kann dazu führen, dass das gesamte System aus der Balance gerät und seine Funktionsfähigkeit (Mittelsysteme) bzw. seine Lebens- und Entwicklungsfähigkeit (lebende Systeme) verliert. Der hier dargestellte Konflikt zwischen Resilienz und Effizienz ist insoweit nur ein Spezialfall einer allgemeinen Gesetzmäßigkeit (vgl. Müller-Reißmann et al. 1988; Müller-Reißmann 1996).

Effizienz - sowohl als Zeit-, wie auch als Kosteneffizienz - ist ,das' Mittel, durch das sich die Rentabilitätsforderungen Geltung verschaffen. Die zentrale Stellung des Rentabilitätsprinzips ist somit maßgeblich für die einseitige Fokussierung auf die Effizienz verantwortlich, wie sie in Wirtschaft, (Wirtschafts-)Wissenschaft und Politik betrieben wird. Denjenigen Arten und Formen, die dem Kriterium der Effizienz nicht in ausreichendem Maße genügen, wird die Daseinsberechtigung versagt (dies gilt nicht nur für ökologische, sondern auch für technologische, kulturelle und soziale Systeme), darunter auch alternative Energien. Sinkt die Vielfalt an Systemelementen aber wegen der einseitigen Betonung des Leitwertes der Effizienz ab, so kann die Funktionsfähigkeit bzw. Lebens- und Entwicklungsfähigkeit der jeweiligen Systeme beeinträchtigt werden. Dies gilt übrigens auch bei einer hypothetischen vollkommenen Internalisierung externer Kosten (also auf Dritte ohne weitere Kompensation abgewälzte Kosten).

Die Überbetonung der Effizienz - im Sinne der weiteren Beförderung der Rentabilität - ist jedoch durchaus im Sinne der herrschenden wirtschaftlichen Interessen. Dies zeigt sich u. a. auch in einer, Gefangennahme' der Politik und der staatlichen Institutionen. Damit werden die Prinzipien der ordoliberalen Schule verworfen (welche u. a. die Idee der ,sozialen Marktwirtschaft' entwickelte): Ihr war die Trennung von privatwirtschaftlichen Interessen und Staat ein Herzensanliegen. Es ging dabei zentral um die Beschränkung von (u. a. wirtschaftlicher) Macht, die quasi als ,kleine Schwester' der Gewalt interpretiert wurde. Nach Eucken ist Macht nicht domestizierbar - es geht daher darum, sie in Wirtschaft und Gesellschaft gar nicht erst entstehen lassen (Eucken 1990). Obwohl der kulturelle und zivilisatorische Fortschritt dahin geführt hat, Gewalt aus Wirtschaft und Gesellschaft zu verbannen und das Gewaltmonopol an den Staat zu geben, wird jedoch (u.a. wirtschaftliche) Macht von weiten Teilen der Gesellschaft als legitim angesehen. Gerade das macht sie aber gefährlich. Nach Eucken sollte einerseits Wirtschaft und Gesellschaft von Macht und andererseits der Staat von privaten Interessen frei gehalten werden. Nur dann ist das Recht in der Lage, eine freiheitliche Ordnung zu garantieren (Eucken 1990). ${ }^{18}$ Jegliche Verquickung von privaten und staatlichen Interessen trägt dazu bei, den Staat zu schwächen. Wenn sich z.B. aus den

\footnotetext{
${ }_{18}$ Ähnliche Gedanken finden sich schon in von Humboldt (2006).
}

Äußerungen des früheren Bundeswirtschaftsministers das Selbstverständnis seines Hauses als verlängerter Arm der Industrielobby erkennen lässt (,wir haben eine wirtschaftsfreundliche Lösung durchgesetzt ${ }^{\circ}$, o. V. 2008), wenn z. B. in Gestalt von durch die Industrie bezahlten ,Leihbeamten“, die an Gesetzen mitwirken, private Interessen unmittelbar in den Staat hineinwirken, wenn ,weiße Korruption' Gang und Gäbe wird und in Gestalt bestimmter Formen des ,Public Private Partnership“ unvereinbare öffentliche Versorgungsund private Renditeinteressen unter das Dach gemeinsamer Unternehmen gesteckt werden, ist diese Trennung nicht mehr vorhanden. All dies trägt dazu bei, auch institutionell die Herrschaft des Rentabilitätsprinzips, die Dominanz der Effizienz zu festigen und letztlich die Lebens- und Entwicklungsfähigkeit unserer Gesellschaft zu gefährden.

Eine Rechtfertigung erfährt die Absolutsetzung der Effizienz auch von der zeitgenössischen Wirtschaftswissenschaft in ihrer herrschenden Form. Insbesondere die neoklassisch basierte Wohlfahrtsökonomie läuft Gefahr, den Wert der Effizienz zu verabsolutieren. Effizienz ist jedoch ein Wert, der Orientierungsrelevanz (wie jeder Wert) nur im ausbalancierten Zusammenspiel mit den anderen Werten besitzt und insofern als Mittel für die Erfüllung der anderen Werte anzusehen ist. Ein zum Selbstzweck überhöhter Wert der Effizienz vermag andere Werte, aufzufressen ' und letzten Endes sogar sich selbst. Eine einseitig effizienzorientierte Strategie zerstört auf die Dauer die Lebens- und Entwicklungsfähigkeit der Gesellschaft, indem sie ihre Vielfalt und Handlungsspielräume, ihre Sicherheit und ihre Gerechtigkeit und damit auch die Quellen ihrer Effizienz untergräbt. Das Effizienzziel sollte ebenfalls nicht dazu missbraucht werden, um durch die Wissenschaft Partikularinteressen $\mathrm{zu}$ verschleiern und ideologisch zu rechtfertigen. Vor diesem Hintergrund wären neue Forschungsfragen zu stellen und ebenso neue institutionelle Designs zu entwickeln (vgl. Löhr 2008). Dem Verfasser ist klar, dass die vorliegenden Gedanken eine Provokation für jeden im Rahmen des herrschenden Paradigmas sozialisierten Ökonomen darstellen. Dennoch sollte die Scientific community den Mut aufbringen, mit dem Primat der Effizienz auch einen Kernsatz ihres Paradigmas (Lakatos 1974) kritisch zu hinterfragen. Normalerweise sterben Paradigmen allerdings erst mit ihren Vertretern; ich bin aber im Zweifel darüber, ob wir genügend Zeit haben, um dies abzuwarten. Daher sollten sich auch Fachwissenschaftler anderer Disziplinen nicht scheuen, kritische Fragen an die (Umwelt-)Ökonomie zu stellen. Möglicherweise steht in den kommenden Jahrzehnten eine Phase dramatischer Herausforderungen bevor (Klimaveränderung, Nahrungsmittelknappheit, Peak Oil etc.), auf welche die (Umwelt-)Ökonomen bislang keine befriedigenden Antworten liefern konnten. Umweltökonomie ist aber zu wichtig, um sie allein den Umweltökonomen zu überlassen. 


\section{Glossar}

Allokation: Hierunter versteht man die ,Kanalisierung ' der knappen Mittel in die effizientesten Verwendungen. Die effiziente Allokation ist ein Hauptanliegen der zeitgenössischen Ökonomie. Allokation ist scharf von Verteilung abzugrenzen.

Barwertkalkül: Bei der Barwertkalkulation werden nicht einfach die Einnahmen und Ausgaben eines Projektes gegenübergestellt, um die Wirtschaftlichkeit zu ermitteln. Vielmehr wird durch ,Diskontierung' berücksichtigt, dass ein Euro, heute eingenommen, mehr wert ist als ein Euro, der z. B. in einem Jahr eingenommen wird. In der Zwischenzeit entgehen nämlich Zinsen $(\rightarrow$ Opportunitätskosten). Entsprechendes gilt mit umgekehrtem Vorzeichen für die Ausgaben.

Cap-and-Trade-System: Es werden begrenzte Rechte an einem Umweltgut definiert, die handelbar gestellt werden. Auch hierbei ist eine Kontrolle notwendig.

Entropie: In der klassischen Thermodynamik ein generelleres Maß für die bei Energieumwandlungen nicht mehr nutzbare Energie.

Kyoto-Regime: Das Kyoto-Protokoll (benannt nach dem Ort der Konferenz Kyōto in Japan) ist ein am 11. Dezember 1997 beschlossenes Zusatzprotokoll zur Ausgestaltung der Klimarahmenkonvention (UNFCCC) der Vereinten Nationen mit dem Ziel des Klimaschutzes. Das am 16. Februar 2005 in Kraft getretene und 2012 auslaufende Abkommen schreibt erstmals völkerrechtlich verbindliche Zielwerte für den Ausstoß von Treibhausgasen fest, welche als hauptsächliche Ursache der globalen Erwärmung angesehen werden. Ein wichtiger Bestandteil des Kyoto-Regimes ist der Emissionshandel, der allerdings nur zwischen Staaten stattfinden soll. Hingegen findet der Emissionshandel auf Europäischer Ebene, der an das Kyoto-Regime ,angedockt' ist, auch zwischen Unternehmen statt.

Leitwerttheorie: Die Leitwerttheorie wurde von Hartmut Bossel begründet. Sie wurde auf den Grundlagen der allgemeinen Systemtheorie entwickelt. Dementsprechend weitgefasst ist ihr Geltungsanspruch. Leitwerte werden hier als Kriterien verstanden. Die ausgewogene Beachtung der sechs von Bossel formulierten Leitwerte (Existenz, Effizienz, Handlungsfreiheit, Sicherheit, Wandlungsfähigkeit und Rücksichtnahme) ist Voraussetzung für die Überlebensfähigkeit und Entwicklungsfähigkeit von Systemen. Bossel sieht das Leitwertgeflecht als flexible, übergeordnete Orientierungsmöglichkeit.

NGO: Non Governmental Organisation/Nicht-Regierungsorganisation

Opportunitätskosten: Die Gewinne, die entgehen, wenn eine bestimmte (Investitions-)Alternative nicht realisiert werden kann, weil man sich für eine andere (Investitions-)Alternative entscheidet.
Resilienz: Elastizität von Ökosystemen.

Sekundärproduktion: Sekundärproduktion ist die Umwandlung von pflanzlicher in tierische Substanz durch Konsumenten.

Utilitarismus: Der utilitaristische Ansatz wurde vor allem durch Jeremy Bentham (1748-1832) und John Stuart Mill (1806-1873) systematisch entwickelt und auf konkrete Fragen angewandt. Er stellt die der herrschenden, neoklassischen Wirtschaftstheorie zugrundeliegende Philosophie dar. Grundlage für die ethische Bewertung einer Handlung ist das Nützlichkeitsprinzip, den Kern des Utilitarismus kann man in der Forderung zusammenfassen: ,Handle so, dass das größtmögliche Maß an Glück entsteht! (Maximum-Happiness-Principle). Die Ökonomie operiert statt ,Glück' mit ,Nutzen‘. Dabei ergibt sich der allgemeine Nutzen aus der Aggregation der Nutzen der einzelnen Individuen. Da mögliche Folgen und reale Wirkungen eine zentrale Stelle in der Beurteilung von Handlungen einnehmen, ist der Utilitarismus eine konsequentialistische Ethik.

Zahlungsbereitschaft: Die Zahlungsbereitschaft ergibt sich aus dem Zahlungswillen (ist also insofern abhängig von den Präferenzen) sowie der Zahlungsfähigkeit (hängt insoweit also vom Budget ab).

Danksagung Für Anregungen danke ich dem Systemforscher Karl Friedrich Müller-Reißmann und Prof. Dr. Eckard Helmers.

\section{Literatur}

SCNAT (Akademie der Naturwissenschaften Schweiz) (2008) Biodiversität und Klima-Konflikte und Synergien im Maßnahmenbereich, Bern

Baumgärtner S, Schiller J (2001) Vielfalt und Nachhaltigkeit-Der Einfluss von Beständen und des Zeithorizonts auf ökonomische Wahlmöglichkeiten. In: Spehl H, Held M (Hrsg), Vom Wert der Vielfalt-Diversität in Ökonomie und Ökologie. Z angew Umweltforsch Sonderh 13:136-147

Borrmann J, Finsinger J (1999) Markt und Regulierung. Vahlen, München

Bossel H (1998) Globale Wende, Wege zu einem gesellschaftlichen und ökologischen Strukturwandel. Droemer Knaur, München

Ciriacy-Wantrup SV (1968) Resource Conservation: economics and politics, $1^{\text {st }}$ edn. Berkeley (University of California Division of Agricultural Science, $3^{\text {rd }}$ edn.).

Costanza R, Cumberland J, Daly H, Goodland R, Norgaard R (2001) Einführung in die Ökologische Ökonomik. Lucius \& Lucius, Stuttgart

Endres A (2000) Umweltökonomie, 2. Aufl. Kohlhammer, Stuttgart u. a.

Eucken W (1990) Grundsätze der Wirtschaftspolitik, 6. Aufl. Mohr Siebeck, Tübingen

Gruhl H (1976) Ein Planet wird geplündert. S. Fischer, Frankfurt a. M.

Henkel HA, Neumann LF, Romahn H (1998) Gegen den gesellschaftspolitischen Imperialismus der reinen Ökonomie. Gedächtnisschrift für Gerhard Weisser. Metropolis-Verlag, Marburg

Hesse H (2003) Ökonomen-Lexikon. Verlag Wirtschaft und Finanzen, Düsseldorf

Keen S (2004) Debunking Economics-The naked emperor of the social sciences. Zed Books Ltd., London/New York 
Kunstler JH (2005) The Long Emergency-Surviving the End of Oil, Climate Change, and Other Converging Catastrophes of the Twenty-First Century. Grove Press, New York

Lakatos I (1974) Falsifikation und die Methodologie wissenschaftlicher Forschungsprogramme. In: Lakatos I, Musgrave A (Hrsg), Kritik und Erkenntnisfortschritt. Vieweg, Braunschweig/Wiesbaden, S. 89-189

Löhr D (2008) Umweltgüter als Common Property Resources. Z UmweltpolitUmweltr 1:49-75

Löhr D (2009) Die Plünderung der Erde-Anatomie einer Ökonomie der Ausbeutung. 2. Aufl. Gauke, Kiel

Meyerhoff J (1999) Ökonomische Bewertung ökologischer Leistungen. 2. Aufl. IÖW, Berlin

Müller-Reißmann KF, Bohmann K, Schaffner J (1988) Kriterien der Sozialverträglichkeit, Studie im Auftrag des Ministers für Arbeit, Gesundheit und Soziales des Landes Nordrhein-Westfalen. Teil A: Theoretische Grundlagen. Hannover

Müller-Reißmann KF (1996) Versuch eines ganzheitlichen Fortschrittsbegriffs. In: Burmeister K, Canzler W, Kalinowski M (Hrsg), Zukunftsfähige Gesellschaft. Verlag Stiftung Mitarbeit, Bonn, S. 35-46

o. V. (2001) Vielseitigkeit ist Trumpf ... Von Pionieren, Kulturfolgern und Generalisten, in: http://www.g-o.de/dossier-detail-99-11. html [16.8.2008] o. V. (2008) Scholz und Glos loben Kompromiss. Focus online, 16. 7.08, in: http://www.focus.de/politik/deutschland/mindestlohn-scholzund-glos-loben-kompromiss_aid_318369.html [16.8.2008]

IVU-Richtlinie (2006): Richtlinie 96/61/EG des Rates vom 24.September 1996 über die integrierte Vermeidung und Verminderung der Umweltverschmutzung (Stand: 18.1.2006)

Scheelhaase J, Grimme W (2006) Emissionshandel für den internationalen Luftverkehr. Z Umweltpolit Umweltr 4:481-501

Secretary of the Convention on Biological Diversity (2001) Handbook of the Convention on Biological Diversity. Earthscan Publications, London

Varela FJ, Maturana HR, Uribe R (1974) Autopoiesis: The organization of living systems, its characterization and a model. Biosyst 5:187-196

von Humboldt W (2006) Ideen zu einem Versuch, die Grenzen der Wirksamkeit des Staats zu bestimmen. Stuttgart

Wiedmann T, Kersten J, Ballschmiter K (2000) Art und Menge von stofflichen Emissionen aus dem Verkehrsbereich-Eine Literaturstudie (Akademie für Technikfolgenabschätzung, Baden-Württemberg). Selbstverlag, Stuttgart

Wuppertal Institut für Klima, Umwelt und Energie (2005) Fair FutureBegrenzte Ressourcen und globale Gerechtigkeit. 2. Aufl. C.H. Beck, München 